\title{
Phytoprotection
}

\section{Pourriture phytophthoréenne de la luzerne au Québec : distribution géographique des symptômes et étude des facteurs édaphiques pouvant favoriser leur expression}

\section{Richard et J.-G. Martin}

Volume 72, numéro 3, 1991

URI : https://id.erudit.org/iderudit/706008ar

DOI : https://doi.org/10.7202/706008ar

Aller au sommaire du numéro

Éditeur(s)

Société de protection des plantes du Québec (SPPQ)l

ISSN

0031-9511 (imprimé)

1710-1603 (numérique)

Découvrir la revue

Citer cet article

Richard, C. \& Martin, J.-G. (1991). Pourriture phytophthoréenne de la luzerne au Québec : distribution géographique des symptômes et étude des facteurs édaphiques pouvant favoriser leur expression. Phytoprotection, 72(3), 87-95. https://doi.org/10.7202/706008ar
Résumé de l'article

Nous avons observé chez la luzerne (Medicago sativa) des symptômes attribuables à la pourriture phytophthoréenne (Phytophthora megasperma f. sp. medicaginis) dans les 12 régions agricoles du Québec, avec une présence plus marquée dans l'Outaouais, la Beauce, les Cantons de l'Est, la région de Québec et le Nord de Montréal. Les symptômes de pourriture étaient plus présents dans les dépressions des luzernières échantillonnées. Nous avons déterminé la texture et la capacité de rétention d'eau des sols échantillonnés, et trouvé aucune relation avec la pourriture phytophthoréenne. La culture de cultivars de luzerne résistants ou sensibles dans ces sols en serre ne montre pas de différence de maladie. Seulement quatre isolats du Phytophthora megasperma f. sp. medicaginis et un du Phytophthora cryptogea ont été obtenus. Lors d'un essai en serre, le trèfle rouge (Trifolium pratense) ne fut pas sensible à l'attaque par le $P$. megasperma f. sp. medicaginis. Le peu de succès à isoler et démontrer la présence du P. megasperma f. sp. medicaginis dans les sols où l'on observe pourtant des symptômes semblables à ceux de la pourriture phytophthoréenne porte à penser qu'en plus du Phytophthora, d'autres causes comme l'anoxie ou l'Aphanomyces euteiches pourraient en être responsables. 


\title{
Pourriture phytophthoréenne de la luzerne au Québec: distribution géographique des symptômes et étude des facteurs édaphiques pouvant favoriser leur expression
}

\author{
C. Richard et J.-G. Martin \\ Station de recherches, Agriculture Canada, 2560, boul. Hochelaga, \\ Sainte-Foy (Québec'), Canada GIV 2J3. Contribution No 420.
}

(Reçu 1991-()4-08; accepté 1991-11-12)

\begin{abstract}
Nous avons observé chez la luzerne (Medicago sativa) des symptômes attribuables à la pourriture phytophthoréenne (Phytophthora megasperma f.sp. medicaginis) dans les 12 régions agricoles du Québec, avec une présence plus marquée dans l'Outaouais, la Beauce, les Cantons de l'Est, la région de Québec et le Nord de Montréal. Les symptômes de pourriture étaient plus présents dans les dépressions des luzernières échantillonnées. Nous avons déterminé la texture et la capacité de rétention d'eau des sols échantillonnés, et trouvé aucune relation avec la pourriture phytophthoréenne. La culture de cultivars de luzerne résistants ou sensibles dans ces sols en serre ne montre pas de différence de maladie. Seulement quatre isolats du Phvtophthora megasperma f. sp. medicaginis et un du Phytophthora cryptogea ont été obtenus. Lors d'un essai en serre, le trèfle rouge (Trifolium pratense) ne fut pas sensible à l'attaque par le $P$. megasperma f. sp. medicaginis. Le peu de succès à isoler et démontrer la présence du $P$. megasperma f. sp. medicaginis dans les sols où l'on observe pourtant des symptômes semblables à ceux de la pourriture phytophthoréenne porte à penser qu'en plus du Phytophthora, d'autres causes comme l'anoxie ou l'Aphanomyces euteiches pourraient en être responsables.
\end{abstract}

Richard, C. et J.-G. Martin. 1991. Pourriture phytophthoréenne de la luzerne au Québec: distribution géographique des symptômes et étude des facteurs édaphiques pouvant favoriser leur expression. PHYTOPROTECTION 72: 87-95.

Symptoms attributed to Phytophthora root rot (Phytophthora megasperma $\mathrm{f}$. sp. medicaginis) were observed on alfalfa (Medicago sativa) in the 12 agronomic regions of the province of Québec; they were more severe in the regions of Outaouais, Beauce, the Eastern Townships, Québec City and the North of Montreal. A greater incidence of the disease was observed in low lying areas. No relationship was found between root rot and water content at field capacity or soil texture. The presence of $P$. megasperma f. sp. medicaginis was not confirmed by the reaction of susceptible and resistant cultivars of alfalfa in greenhouse assays. Only four isolates of Phytophthora megasperma f. sp. medicaginis and one of Phytophthora cryptogea were obtained. Red clover (Trifolium pratense) was not affected by P. megasperma f. sp. medicaginis in a greenhouse trial. The difficulty to detect and isolate Phytophthora in soil samples led to the conclusion that other factors such as anoxia or Aphanomyces euteiches might also be involved.

\section{Introduction}

Le Phytophthoramegasperma Drechs. f.sp. medicaginis T. Kuan \& D.C. Erwin (Pmm) attaque les racines de la luzerne (Medicago sativa L.) dans les sols mal drainés ou dans les endroits où la nappe phréatique est près de la surface, maintenant ainsi le sol saturé d'eau durant une période prolongée (Kuan et Erwin 1980b). Le Pmm est un parasite peu compétitif et difficile à isoler en présence de Pythium et de Fusarium (Erwin 1954; Schmitthenner 1964). Quoique les symptômes de cette pourriture furent observés depuis longtemps au Québec, le P.megaspermaf.sp.medicaginis ne fut isolé du sol de luzernières de l'ouest du Québec et de l'Ontario que récemment (Faris et Sabo 1981), et des racines de la luzerne pour la première fois en 1985 (Richard 1985). Il s'agissait du type à petites spores du Pmm, plus virulent pour la luzerne que le type à larges oogones (Richard 1985).

$\overline{0031-9511 / 91 \$ 1.00+.10}$
On connaît peu l'importance de cette maladie et les facteurs qui influencent sa présence et son développement dans les légumineuses fourragères au Québec. De plus, la sursaturation des sols en eau et d'autres champignons pathogènes peuvent causer des dommages difficilement séparables du Pmm (Alva et al. 1985). Les buts du présent travail sont donc d'établir la distribution géographique des symptômes du Pmm dans les luzernières du Québec, d'isoler le Pmm des sols et des plantes échantillonnés dans chacune des régions, d'identifier les facteurs édaphiques pouvant favoriser la présence de symptômes de la pourriture phytophthoréenne, et d'établir la sensibilité du trèfle rouge (Trifolium pratense L.) au $P$. megasperma f. sp. medicaginis.

\section{Matériel et méthodes}

Échantillonnage. L'échantillonnage couvre 46 comtés répartis dans les 12 régions agricoles du Québec à raison d'une luzernière par environ 4000 ha en foin de luzerne. Des 
plantes et des échantillons de sol se trouvant dans leur environnement ont été prélevés dans chacune des régions agricoles en juin et août 1985. Soixante-quatre luzernières en production ( $2^{\mathrm{e}}$ année et plus), d'environ 10 ha et dont 41 présentaient de la mortalité dans les dépressions, ont été visitées (tableau 1).

Nous avons localisé quatre emplacements dans chaque luzernière et nous avons noté le recouvrement visuel de la luzerne $(1=$ sporadique; 2 =clairsemée; $3=$ dense); dans un rayon de $5 \mathrm{~m}$, nous avons prélevé cinq plantes avec leur système racinaire pour déceler des symptômes de pourriture. Pour les 41 luzernières qui présentaient de la mortalité dans les dépressions, les emplacements ont été distribués à raison de deux dans des dépressions et deux sur des buttes. Les échantillons ont été placés dans des glacières pour le transport et ensuite conservés en chambre froide.

L'indice de pourriture (IP) est déterminé pour chaque plante selon une échelle de 1 à 6 utilisée pour la pourriture phytophthoréenne $(1=$ aucun symptôme, radicelles intactes; $2=$ pas de lésion sur la racine principale, mais la plupart des radicelles détruites, laissant un point noir au point d'attachement; $3=$ =plages noires sur la racine principale ou sur une ou deux racines secondaires; $4=$ une partie de la racine principale détruite; $5=$ presque toute la racine principale détruite; $6=$ plante morte) (Faris et al. 1983; Frosheiser et Barnes 1973). L'indice des symptômes du feuillage (ISF) est aussi déterminé pour chaque plante selon une échelle de 1 à 4 ( 1 = aucun symptôme; 2 = chlorose de deux ou trois feuilles; $3=$ chlorose générale; $4=$ plante morte).

Dans chaque luzernière, nous avons aussi recueilli du sol à deux emplacements (une butte et une dépression) pour tenter d'en isoler le Pmmet pouren déterminer la granulométrie par la méthode de l'hydromètre (Bouyoucos 1936) et la capacité de rétention d'eau par la méthode del'humidité équivalente de Bouyoucos (Hanks et al. 1954). Les classes texturales des sols ont été déterminées à l'aide de l'abaque de la Classification canadienne des sols (Jacob et al. 1989).

Détection du Pmm dans les sols échantillonnés. Cette expérience a été effectuée avec deux séries de 40 sols provenant des buttes et des dépressions (le sol de la région de la Beauce est manquant). Des plantes de luzerne (un cul- tivar sensible, Saranac, et un résistant, 120) ont été cultivées en serre dans chaque sol provenant des différents lieux d'échantillonnage afin de détecter, par la réaction différentielle des cultivars, la présence du Pmm dans les sols échantillonnés. La luzerne a été semée par paire (le cultivar sensible et le résistant) dans des pots de $15 \mathrm{~cm}$ (un pot par sol) de façon à avoir 10 plantes du cultivar résistant d'un côté du pot et 10 du cultivar sensible de l'autre (Faris et al. 1983). Après 21 jours de croissance, l'apport en eau est augmenté afin de saturer le sol 4 jours sur 7 pour une période de 21 jours (Kuan et Erwin 1980a), à $25^{\circ} \mathrm{C}$ pour une photopériode de $16 \mathrm{~h}[350$ à $\left.400 \mu \mathrm{mol} /\left(\mathrm{m}^{\prime} \cdot \mathrm{s}\right)\right]$ et à $20^{\circ} \mathrm{C}$ pour les $8 \mathrm{~h}$ de noirceur. Nous avons alors déterminé l'IP, l'ISF et la masse de matière sèche (MS) par plante pour chaque cultivar. Les plantules de luzerne servaient également de plantes-pièges pour l'isolement du Pmm (Marks et Mitchell 1970) en vue de détecter sa présence dans les sols ou de compléter la vérification du postulat de Koch dans le cas du pouvoir pathogène.

Isolement du Pmm. Nous avons désinfecté des segments de racines prélevés près des nécroses avec de l'hypochlorite de sodium $(0,5 \%)$, que nous avons ensuite rincées à l'eau stérile et ensemencées sur un milieu gélosé à base de jus de légumes V-8 (4\%) contenant cinq substances antifongiques (pentachloronitrobenzène, bénomyl, néomycine, rifampicine et hymexazol) (Canaday et Schmitthenner 1982). Les champignons isolés ont ensuite été repiqués sur du jus de légumes V-8 (20\%) gélosé additionné de $2 \%$ de $\mathrm{CaCO}_{3}$ (Marks et Mitchell 1970) et identifiés.

Évaluation de substrats. En vue des expériences sur la sensibilité du trèfle rouge au Pmm et de la sélection génétique de la luzerne pour la résistance au Pmm, nous avons testé quatre substrats de croissance stérilisés à l'autoclave pour déterminer celui qui favorise le plus l'expression des symptômes de la pourriture phytophthoréenne: un terreau (sable, $40 \%$; limon, $25 \%$; argile, $20 \%$; matière organique, $15 \%$ ), le Redi-Earth (mousse de tourbe, $50 \%$; vermiculite, $50 \%$ ), le Turface (argile montmorillonite calcifiée) et la silice. Nous y avons comparé en cabinet de croissance les cultivars de luzerne Agate (résistant) et Saranac (sensible) inoculés avec deux souches connues provenant de régions géographiques différentes, la souche 325 isolée d'une racine de luzerne malade provenant de Masson, comté de Papineau, et la sou- 
che 270, isolée d'un sol de luzernière de SaintCésaire, comté de Rouville (gracieusement fournie par F.E. Sabo du Centre de recherches en phytotechnie, Ottawa). Nous avons préparé l'inoculum en broyant, dans un mélangeur, le contenu de deux cultures âgées de 6 jours $\left(22^{\circ} \mathrm{C}\right.$, noirceur) en boîte de Pétri avec $1 \mathrm{Ld}$ 'eau distillée stérile. Nous avons versé $50 \mathrm{~mL}$ d'inoculum dans un sillon le long de chaque rang de luzerne âgée de 21 jours (Kuan et Erwin 1980a). L'expérience a été réalisée selon le même protocole que celui utilisé pour la détection du Pmm dans les sols sauf pour le nombre de plantes par pot qui fut de cinq par cultivar. L'IP, l'ISF et la MS ont été déterminés pour chacun des cultivars selon chaque souche et chaque substrat.

Sensibilité du trèfle rouge au $P$. megasperma f. sp. medicaginis. Nous avons testé la sensibilité du trèfle rouge au Pmm (souches 270 et 325) avec les cultivars Lakeland (semi-tardif) et Ottawa (hâtif); les cultivars de luzerne Apollo (résistant) et Saranac (sensible) ont servi de référence.

L'expérience a été réalisée dans du terreau selon le même protocole que celui utilisé pour l'évaluation des substrats. L'IP, l'ISF et la MS ont été déterminés pour chacun des cultivars, selon chaque souche et chaque substrat.
Dispositifs expérimentaux et analyses statistiques. Sauf pour le test dans les sols de luzernière dans lequel chaque sol n'est représenté qu'une seule fois, les expériences ont été effectuées en cinq répétitions (quatre pourl'étude des substrats) selon un dispositif en tiroir dans lequel le pot est la parcelle principale et le cultivar, la sous-parcelle. Une analyse de variance (tests par paires de cultivars sur les sols prélevés et sur les substrats) suivie d'un test de Duncan (texture des sols, substrats) ou un test de $t$ (indice de pourriture et recouvrement entre buttes et dépression, pouvoir pathogène) ont été utilisés pour analyser les résultats (SAS Institute Inc. 1985b). Le coefficient de corrélation linéaire de Pearson a été déterminé pour établir la relation entre la présence des symptôme de Pmm au champ et la capacité de rétention d'eau de leur sol (SAS Institute Inc. 1985a).

\section{Résultats et discussion}

Distribution géographique des symptômes du Pmm. Nous avons observé des symptômes de pourriture pouvant être attribués au Pmm dans toutes les régions et dans la presque totalité des 64 luzernières échantillonnées. La pourriture a été qualifiée de grave (IP de 3 et plus) à au moins un emplacement dans 18 luzernières. Les indices de pourriture moyens les plus élevés ont été observés dans les régions

Tableau 1. Gravité de la pourriture phytophthoréenne de la luzerne dans les 12 régions agricoles du Québec

\begin{tabular}{lcc}
\hline Région & Nombre de luzernières & IP $^{\S}$ \\
\hline Bas du fleuve & 6 & 1,59 \\
Québec & 7 & 2,26 \\
Beauce & 1 & 2,95 \\
Nicolet & 6 & 1,55 \\
Cantons de l'Est & 1 & 2,50 \\
Est de Montréal & 12 & 1,44 \\
Sud-ouest de Montréal & 7 & 1,50 \\
Outaouais & 7 & 2,43 \\
Abitibi & 3 & 1,60 \\
Nord de Montréal & 7 & 2,27 \\
Mauricie & 3 & 1,49 \\
Saguenay - Lac Saint-Jean & 4 & 1,51 \\
Total & 64 & 1,92 \\
\hline
\end{tabular}

$\S$ Indice de pourriture phytophthoréenne (1= aucun symptôme, radicelles intactes; $2=$ pas de lésion sur la racine principale, mais la plupart des radicelles détruites, laissant un point noir au point d'attachement; $3=$ plages noires sur la racine principale ou sur une ou deux racines secondaires, $4=$ une partie de la racine principale détruite; $5=$ presque toute la racine principale détruite; $6=$ plante morte). 
de la Beauce, des Cantons de l'Est, de l'Outaouais, du Nord de Montréal et de Québec (tableau 1). C'est également à partir de sols de l'Outaouais et de la région de Québec, en plus de l'Abitibi, que nous avons réussi à isoler le Pmm. Des 64 luzernières échantillonnées, seulement quatre isolats du Pmm ont été obtenus (6\% des luzernières). Ce faible pourcentage d'isolement est du même ordre de grandeur que celui trouvé au Wisconsin (Pratt et Mitchell 1973), mais très inférieur à ce qui a été trouvé en Ontario (Chi 1970). Cependant, les Phytophthora que nous avons isolés provenaient tous de sols dans lesquels les plantules de luzerne servant de plantes-pièges avaient un IP très élevé (4,3 en moyenne). Nous avons aussi isolé le Phytophthora cryptogea Pethybr. \& Lafferty. C'est un autre Phytophthora pathogène qui affectionne les endroits mal drainés et qui a été signalé chez la luzerne au Japon (Matsumoto et Sato 1979).

\section{Facteurs édaphiques pouvant favoriser la présence des symptômes attribués au Pmm. Le degré de pourriture (IP) moyen pour les luzernièreséchantillonnées était plus élevé dans les dépressions des champs où l'eau libre a}

tendance à s'accumuler (tableau 2), ce qui correspond à la niche écologique du Pmm (Erwin 1990). Cette différence de pourriture entre les buttes et les dépressions était significative dans les régions de Québec et de la Beauce $(P \leq 0,05)$. $C$ 'est aussi dans les dépressions que nous avons observé le recouvrement le plus faible dans cinq régions et pourl'ensemble des régions $(P \leq 0,01)$ (tableau 2) vraisemblablement dû à la mortalité de la luzerne qu'on impute généralement au mauvais drainage. Cette mortalité pourrait aussi être reliée à la pourriture phytophthoréenne puisque l'on y trouve, au printemps et lors des pluies abondantes, l'eau libre nécessaire à la dissémination des zoospores du Pmm. La saturation du sol en eau prédispose la luzerne à l'attaque par le Pmm (Kuan et Erwin 1980b).

L'analyse granulométrique a permis de classer les 125 échantillons de sols en 11 classes texturales (tableau 3). Leur capacité de rétention d'eau décroît avec la grosseur des particules. Quoiqu'on observe des différences de rétention d'eau significatives entre certaines classes texturales, nous n'avons observé aucune relation significative entre la pourriture et la capacité de rétention d'eau $(r=0,103)$.

Tableau 2. Gravité de la pourriture phytophthoréenne et densité de la luzerne selon la topographie du terrain et les régions agricoles du Québec

\begin{tabular}{|c|c|c|c|c|c|c|c|}
\hline \multirow[b]{2}{*}{ Région } & \multirow{2}{*}{$\begin{array}{l}\text { Nombre de } \\
\text { luzernières }\end{array}$} & \multicolumn{3}{|c|}{$I^{\$}$} & \multicolumn{3}{|c|}{ Recouvrement de la luzerne } \\
\hline & & Butte & Dépression & Différence & Butte & Dépression & Différence \\
\hline Bas du fleuve & 4 & 1,61 & 1,51 & $-0,10$ & 2,50 & 2,00 & 0,50 \\
\hline Québec & 4 & 1,48 & 2,65 & $1,17^{*}$ & 2,50 & 1,50 & $1,00 * *$ \\
\hline Beauce & 1 & 2,20 & 3,70 & $1,50^{*}$ & 3,00 & 1,50 & 1,50 \\
\hline Nicolet & 5 & 1,59 & 1,47 & $-0,12$ & 2,60 & 1,60 & $1,00 * *$ \\
\hline Cantons de l'Est & 1 & 2,10 & 2,90 & 0,80 & 2,00 & 1,00 & $1,00 * * *$ \\
\hline Est de Montréal & 9 & 1,36 & 1,51 & 0,15 & 2,61 & 2,11 & $0,5)^{*}$ \\
\hline Sud-ouest de Montréal & 4 & 1,63 & 1,75 & 0,12 & 2,25 & 1,50 & 0,75 \\
\hline Outaouais & 4 & 2,88 & 2,73 & $-0,15$ & 2,38 & 1,75 & 0,63 \\
\hline Abitibi & 1 & 1,90 & 1,40 & $-0,50$ & 1,50 & 1,00 & 0,50 \\
\hline Nord de Montréal & 3 & 2,50 & 2,40 & $-0,10$ & 2,83 & 1,83 & $1,00 *$ \\
\hline Mauricie & 3 & 1,32 & 1,67 & 0,35 & 2,83 & 2,00 & 0,83 \\
\hline Saguenay-Lac Saint-Jean & 2 & 1,90 & 1,60 & $-0,30$ & 2,50 & 2,00 & 0,50 \\
\hline Moyenne & 41 & 1,87 & 2,11 & 0,24 & 2,46 & 1,65 & $0,81 * *$ \\
\hline
\end{tabular}

\footnotetext{
$\S$ Indice de pourriture phytophthoréenne (1= aucun symptôme, radicelles intactes; $2=$ pas de lésion sur la racine principale, mais la plupart des radicelles détruites, laissant un point noir au point d'attachement; $3=$ plages noires sur la racine principale ou sur une ou deux racines secondaires; $4=$ une partie de la racine principale détruite; $5=$ presque toute la racine principale détruite; $6=$ plante morte).

$\dagger 1$ = sporadique; 2 =clairsemée; $3=$ dense.

† Dépression moins butte.

*, Significatif à $P \leq 0,05 ; * *$, significatif à $P \leq 0,01$.
} 
Tableau 3. Intensité des symptômes de la pourriture phytophthoréenne et capacité de rétention d'eau selon la texture des sols

\begin{tabular}{lccc}
\hline & & \multicolumn{2}{c}{ Capacité de rétention d'eau } \\
\cline { 3 - 4 } Classe & $\begin{array}{c}\text { Nombre } \\
\text { d'échantillons }\end{array}$ & $(\%)$ & $\mathrm{IP}^{\S}$ \\
\hline Argile limoneuse & 2 & $47,0 \mathrm{a}^{\dagger}$ & $1,9 \mathrm{ab}$ \\
Argile & 4 & $46,8 \mathrm{a}$ & $1,8 \mathrm{ab}$ \\
Argile lourde & 15 & $46,0 \mathrm{a}$ & $1,7 \mathrm{ab}$ \\
Loam limono-argileux & 24 & $41,0 \mathrm{ab}$ & $1,6 \mathrm{~b}$ \\
Loam argileux & 2 & $40,0 \mathrm{ab}$ & $2,0 \mathrm{ab}$ \\
Loam sablo-argileux & 10 & $39,3 \mathrm{ab}$ & $2,3 \mathrm{ab}$ \\
Loam limoneux & 21 & $37,3 \mathrm{abc}$ & $2,8 \mathrm{a}$ \\
Loam & 4 & $34,2 \mathrm{bc}$ & $2,0 \mathrm{ab}$ \\
Loam sableux & 4 & $30,6 \mathrm{bc}$ & $1,4 \mathrm{~b}$ \\
Sable & 27 & $28,8 \mathrm{c}$ & $2,2 \mathrm{ab}$ \\
Sable loameux & 12 & $26,9 \mathrm{c}$ & $1,4 \mathrm{~b}$ \\
\hline
\end{tabular}

$\S$ Indice de pourriture phytophthoréenne (1= aucun symptôme, radicelles intactes; $2=$ pas de lésion sur la racine principale. mais la plupart des radicelles détruites, laissant un point noir au point d'attachement; $3=$ plages noires sur la racine principale ou sur une ou deux racines secondaires; $4=$ une partie de la racine principale détruite; $5=$ presque toute la racine principale détruite; $6=$ plante morte).

$\doteqdot$ Dans une même colonne, les moyennes suivies d'une même lettre ne sont pas significativement différentes selon le test de Duncan $(P>0,05)$.

Une part des symptômes de pourriture observés au champ sont peut-être dus aussi à un manque d'oxygène (anoxie) causé par un excès d'eau (Knous et Maxfield 1976) ou à un autre champignon pathogène (Havey et Grau 1985). Les dommages dus au Pmm et ceux dus à l'eau en excès dans le sol sont difficiles à séparer (Alva et al. 1985). Dans une autre étude de luzernières mal drainées où la luzerne survivait mal, nous avons isolé du sol l'Aphanomyces euteiches Drechs. (Richard et al. 1991). L'A. euteiches attaque la luzerne de la même façon

Tableau 4. Analyse de variance de l'ISF, de l'IP et de la MS de chaque pot contenant un cultivar de luzerne sensible (Saranac) et un cultivar résistant (120) cultivés dans les sols prélevés sur une butte et une dépression de chaque luzernière échantillonnée

\begin{tabular}{lllc}
\hline Facteur & $\mathrm{ISF}^{\S}$ & $\mathrm{IP}^{\dagger}$ & MS (mg/plante) \\
\hline Emplacement (E) & & & \\
$\quad$ Butte & 2,03 & 2,49 & 36 \\
$\quad$ Dépression & 2,21 & 2,80 & 31 \\
$\quad P$ & 0,1349 & 0,1054 & 0,0831 \\
Cultivar (C) & & & 31 \\
$\quad$ Résistant (120) & 2,08 & 2,57 & 36 \\
$\quad$ Sensible (Saranac) & 2,17 & 2,71 & 0,0151 \\
$P$ & 0,2818 & 0,2209 & 0,1611 \\
C $\times$ E & & & 44,6 \\
$\quad P$ & 0,8852 & 0,3438 & 49,2 \\
Coefficient de variation & 30,7 & 39,2 & \\
\hline
\end{tabular}

$\S$ Indice des symptômes du feuillage ( $1=$ aucun symptôme; $2=$ chlorose de deux ou trois feuilles; $3=$ chlorose générale; $4=$ plante morte).

$\doteqdot$ Indice de pourriture phytophthoréenne ( $1=$ aucun symptôme, radicelles intactes; $2=$ pas de lésion sur la racine principale, mais la plupart des radicelles détruites, laissant un point noir au point d'attachement; $3=$ plages noires sur la racine principale ou sur une ou deux racines secondaires; $4=$ une partie de la racine principale détruite; $5=$ presque toute la racine principale détruite; $6=$ plante morte). 
que le Pmm et cause des dégâts semblables (Delwiche et al. 1987; McKeen et Traquair 1980) dans les mêmes conditions de mauvais drainage du sol, mais les symptômes sur les plantes adultes sont moins bien définis que ceux de la pourriture phytophthoréenne avec laquelle ils peuvent être confondus (Grau 1990). C'est peut-être pour cette raison que nous avons isolé si peu de Pmm, malgré la fréquence élevée d'observation des symptômes attribués à la pourriture phytophthoréenne.

Détection du Pmm dans les sols. L'analyse de variance du test de comparaison des deux cultivars (tableau 4) ne montre pas de différence de maladie entre les buttes et les dépressions; la comparaison du cultivar sensible et du résistant n'a pas non plus montré de différence de maladie, mais seulement une différence au niveau du rendement en matière sèche en faveur du cultivar sensible.

Évaluation desubstrats. La différence entre les substrats est très significative pour les rendements en matière sèche $(P=0,0001)$ et l'ISF $(P=0,0018)$ (tableau 5). Dans le Turface et dans la silice, le rendement de la luzerne n'a été que d'environ $30 \%$ et de $50 \%$, respectivement, de celui obtenu dans le terreau et dans le RediEarth .

Des deux souches testées, c'est la souche 325 qui s'est avérée la plus virulente, donnant

Tableau 5. Analyse de variance de l'ISF, de l'IP et de la MS par substrat par souche de Phytophthora megasperma f. sp. medicaginis et par cultivar de luzerne

\begin{tabular}{|c|c|c|c|}
\hline Facteur & ISF $^{\S}$ & IP" & MS (mg/plante) \\
\hline \multicolumn{4}{|l|}{ Substrat (S) } \\
\hline Turface & $2,01 a^{\ddagger}$ & 2,68 & $86 \mathrm{~b}$ \\
\hline Silice & $1,98 \mathrm{a}$ & 2,77 & $126 \mathrm{~b}$ \\
\hline Terreau & $1,54 \mathrm{~b}$ & 2,39 & $277 a$ \\
\hline Redi Earth & $1,41 \mathrm{~b}$ & 2,52 & $243 a$ \\
\hline$P$ & 0,0018 & 0,5801 & 0,0001 \\
\hline \multicolumn{4}{|l|}{ Souche (I) } \\
\hline 270 & 1,50 & 2,18 & 177 \\
\hline 325 & 1,97 & 3,00 & 190 \\
\hline$P$ & 0,0006 & 0,0003 & 0,5594 \\
\hline \multicolumn{4}{|l|}{ Cultivar $(\mathrm{C})$} \\
\hline Résistant (Agate) & 1,52 & 2,06 & 190 \\
\hline Sensible (Saranac) & 1,95 & 3,12 & 176 \\
\hline$P$ & 0,0079 & 0,0185 & 0,6924 \\
\hline \multicolumn{4}{|l|}{$\mathrm{S} \times \mathrm{I}$} \\
\hline$P$ & 0,5255 & 0,6763 & 0,1029 \\
\hline \multicolumn{4}{|l|}{$\mathrm{S} \times \mathrm{C}$} \\
\hline$P$ & 0,0597 & 0.5491 & 0,3581 \\
\hline \multicolumn{4}{|l|}{$\mathrm{I} \times \mathrm{C}$} \\
\hline$P$ & 0,6264 & 0,0602 & 0,3202 \\
\hline \multicolumn{4}{|l|}{$\mathrm{S} \times \mathrm{I} \times \mathrm{C}$} \\
\hline$P$ & 0,1947 & 0,6336 & 0,8918 \\
\hline Coefficient de variation & 28,5 & 31,9 & 48,2 \\
\hline
\end{tabular}

$\S$ Indice des symptômes du feuillage ( $I=$ aucun symptôme; $2=$ chlorose de deux ou trois feuilles; $3=$ chlorose générale; 4= plante morte).

$\dagger$ Indice de pourriture phytophthoréenne (1= aucun symptôme, radicelles intactes; $2=$ pas de lésion sur la racine principale, mais la plupart des radicelles détruites, laissant un point noir au point d'attachement; $3=$ plages noires sur la racine principale ou sur une ou deux racines secondaires; $4=$ une partie de la racine principale détruite; $5=$ presque toute la racine principale détruite; $6=$ plante morte).

† Dans une même colonne, les moyennes suivies d'une mème lettre ne sont pas significativement différentes selon le test de Duncan $(P>0,05)$ 
Tableau 6. ISF, IP et MS d'un cultivar de luzerne sensible (Saranac) et d'un cultivar résistant (Agate) cultivés dans quatre substrats et inoculés avec deux souches de Phytophthora megasperma f. sp. medicaginis

\begin{tabular}{|c|c|c|c|c|c|}
\hline Substrat & Souche & Cultivar & ISF $^{\S}$ & $\mathrm{IP}^{\dagger}$ & MS (mg/plante) \\
\hline \multirow[t]{4}{*}{ Terreau } & \multirow[t]{2}{*}{270} & Agate & 1,25 & 1,63 & 246 \\
\hline & & Saranac & 1,33 & 2,25 & 197 \\
\hline & \multirow[t]{2}{*}{325} & Agate & 1,40 & 1,80 & 368 \\
\hline & & Saranac & 2,17 & 3,88 & 295 \\
\hline \multirow[t]{4}{*}{ Redi-Earth } & \multirow[t]{2}{*}{270} & Agate & 1,06 & 1,86 & 209 \\
\hline & & Saranac & 1,06 & 2,13 & 309 \\
\hline & \multirow[t]{2}{*}{325} & Agate & 1,53 & 2,43 & 231 \\
\hline & & Saranac & 2,00 & 3,65 & 225 \\
\hline \multirow[t]{4}{*}{ Turface } & \multirow[t]{2}{*}{270} & Agate & 1,29 & 1,73 & 110 \\
\hline & & Saranac & 2,23 & 2,67 & 89 \\
\hline & \multirow[t]{2}{*}{325} & Agate & 1,73 & 2,24 & 100 \\
\hline & & Saranac & 2,79 & 4,08 & 49 \\
\hline \multirow[t]{4}{*}{ Silice } & \multirow[t]{2}{*}{270} & Agate & 1,67 & 2,17 & 124 \\
\hline & & Saranac & 2,13 & 3,00 & 131 \\
\hline & \multirow[t]{2}{*}{325} & Agate & 2,23 & 2,58 & 133 \\
\hline & & Saranac & 1,89 & 3,33 & 117 \\
\hline
\end{tabular}

$\S$ Indice des symptômes du feuillage ( $1=$ aucun symptôme; $2=$ chlorose de deux ou trois feuilles; $3=$ chlorose générale; $4=$ plante morte).

$\doteqdot$ Indice de pourriture phytophthoréenne ( $1=$ aucun symptôme, radicelles intactes; $2=$ pas de lésion sur la racine principale, mais la plupart des radicelles détruites, laissant un point noir au point d'attachement; $3=$ plages noires sur la racine principale ou sur une ou deux racines secondaires; $4=$ une partie de la racine principale détruite; $5=$ presque toute la racine principale détruite; $6=$ plante morte).

un $\operatorname{ISF}(P=0,0006)$ et un $\operatorname{IP}(P=0,0003)$ plus élevés (tableau 5) quels que soient le substrat et le cultivar sauf l'ISF de 'Saranac' dans la silice (tableau 6). Faris et al. (1983) ont aussi trouvé que le pouvoir pathogène pouvait être très différent d'une souche à l'autre.

La méthode utilisée a permis de distinguer le cultivar sensible du cultivar résistant au Pmm en ce qui a trait à l'ISF $(P=0,0079)$ et à l'IP $(P=0,0185)$.

Comme la pourriture phytophthoréenne est une maladie essentiellement racinaire et que c'est le terreau qui a permis d'obtenir la plus forte croissance de la luzerne et d'observer la plus grande différence d'IP $(2,08)$ entre le cultivar résistant et le cultivar sensible, pour la souche la plus virulente (325) (tableau 6), il semble que ce substrat soit préférable pour les expériences avec la pourriture phytophthoréenne.
Pathogénicité envers le trèfle rouge et la luzerne. Le Pmm inoculé au trèfle rouge n'a causé aucun symptôme sur le feuillage ni sur les racines, et n'a pas eu d'effet sur sa croissance alors que sur la luzerne, il a provoqué des symptômes très marqués à des niveaux significativement différents $(P \leq 0,01)$ entre le cultivar sensible et le cultivar résistant (tableau 7). Ces résultats confirment que le Pmm est spécifique à la luzerne (Kuan et Erwin 1980a) et que le trèfle rouge est résistant au Pmm (Pratt 1981). Cependant, une souche de la luzerne du $P$. megasperma Drechs. a provoqué une forte fonte de semis chez les mêmes cultivars de trèfle rouge (Chi 1966), mais la fonte de semis n'est pas un critère valable pour établir la gamme d'hôtes d'un champignon pathogène (Pratt 1981). Le Phytophthora megasperma f. sp. trifolii Pratt et le Phytophthora erythroseptica Pethybr. sont par contre pathogènes pour le Trifolium vesiculosum Savi (Pratt 1981). 
Tableau 7. Pouvoir pathogène du Phytophthora megasperma f. sp. medicaginis envers le trèfle et la luzerne élevés dans un terreau en conditions d'inondation

\begin{tabular}{|c|c|c|c|c|}
\hline Cultivar & Traitement & ISF $^{\lessgtr}$ & $\mathrm{IP}^{t}$ & MS (g/plante) \\
\hline \multicolumn{5}{|l|}{ Luzerne } \\
\hline \multirow[t]{2}{*}{ Apollo } & Inoculé & 2,4 ** * & $3,3 * *$ & 0,55 \\
\hline & Non inoculé & 1,0 & 1,2 & 0,68 \\
\hline \multirow[t]{2}{*}{ Saranac } & Inoculé & $3,3 * *$ & $4,2 * * ;$ & 0,56 \\
\hline & Non inoculé & 1,0 & 1,2 & $(0,66$ \\
\hline \multicolumn{5}{|l|}{ Trèfle } \\
\hline \multirow[t]{2}{*}{ Lakeland } & Inoculé & $1,()$ & 1,1 & 0,90 \\
\hline & Non inoculé & 1,0 & 1,1 & 0,96 \\
\hline \multirow[t]{2}{*}{ Ottawa } & Inoculé & 1,0 & 1,1 & 1,20 \\
\hline & Non inoculé & 1,0 & 1,1 & 1,16 \\
\hline
\end{tabular}

$\$ \quad$ Indice des symptômes du feuillage ( $1=$ aucun symptôme; $2=$ chlorose de deux ou trois feuilles; $3=$ chlorose générale; $4=$ plante morte).

* Indice de pourriture phytophthoréenne ( $1=$ aucun symptôme, radicelles intactes; $2=$ pas de lésion sur la racine principale, mais la plupart des radicelles détruites, laissant un point noir au point d'attachement; $3=$ plages noires sur la racine principale ou sur une ou deux racines secondaires; $4=$ une partie de la racine principale détruite; $5=$ presque toute la racine principale détruite; $6=$ plante morte).

**, Significativement différent du témoin non inoculé correspondant, à $P<0,01$.

\section{Conclusions}

En général au Québec, les symptômes attribués à la pourriture phytophthoréenne de la luzerne sont plus graves dans les dépressions du champ, sans doute à cause de la disponibilité de l'eau au printemps ou lors de pluies abondantes. Elle n'est cependant pas reliée à la capacité de rétention d'eau des sols. La présence des symptômes est donc reliée à la topographie du terrain plutôt qu'à la texture du sol. A cause du faible taux d'isolement du Pmm et de la faible différence entre les cultivars sensibles et résistants dans les sols prélevés, nous pensons que les symptômes observés au champ n'ont pas toujours été causés seulement par le Pmm, mais peuvent aussi avoir été la résultante de l'action de plusieurs causes comme l'anoxie et, en plus du Pmm, un autre champignon pathogène, l'Aphanomyces euteiches. Les souches de Pmm testés ne sont pas pathogènes pour les cultivars de trèfle rouge testés.

Les auteurs remercient Carole Gauvin-Trudel et Léon Lambert pour leur aide technique.

Alva, A.K., L.E. Lanyon et K.T. Leath. 1985. Excess soil water and Phytophthora root rot stresses of Phytophthora root rot sensitive and resistant alfalfa cultivars. Agron. J. 77: 437-442.
Bouyoucos, G.J. 1936. Directions for making mechanical analysis of soils by the hydrometer method. Soil Sci. 42: 225-229.

Canaday, C.H. et A.F. Schmitthenner. 1982. Isolating Phvtophthora megasperma f. sp. glycinea from soil with a baiting method that minimizes Pythium contamination. Soil Biol. Biochem. 14: 67-68.

Chi, C.C. 1966. Phytophthora root rot of alfalfa in Canada. Plant Dis. Rep. 50: 451-453.

Chi, C.C. 1970. Phytophthora root rot of alfalfa in Ontario in 1969. Can. Plant Dis. Surv. 50: 109-110.

Delwiche, P.A., C.R. (Grau, E.B. Holub et J.B. Perry. 1987. Characterization of Aphanomyce's euteiches isolates recovered from alfalfa in Wisconsin. Plant Dis. 71: 155-161.

Erwin, D.C. 1954. Root rot of alfalfa caused by $P h y$ tophthora (ryptogea. Phytopathology $44: 700-7(04$.

Erwin, D.C. 1990. Phytophthora root rot. Pages 37-39 in D.L. Stuteville et D.C. Erwin (réd.), Compendium of alfalfa diseases. American Phytopathological Society. St. Paul.

Faris, M.A. et F.E. Sabo. 1981. Progress report on pathogenicity differential studies of 74 Phytophthora megasperma Drechsler isolates from alfalfa. Forage Notes 25(2): 23-30.

Faris, M.A., F.E.Sabo et D.J.S. Barr. 1983. Studies on Phytophthora megasperma isolates with different levels of pathogenicity on alfalfa cultivars. Can J. Plant Pathol. 5: 29-33.

Frosheiser, F.I. et D.K. Barnes. 1973. Field and greenhouse selection for Phytophthora root rot resistance in alfalfa. Crop Sci. 13: 735-738.

Grau, C. R. 1990. A phanomyces root rot. Pages 10-11 in D.L. Stuteville et D.C. Erwin (réd.), Compendium of alfalfa diseases. American Phytopathological Society, St. Paul. 
Hanks, R.J., W.E. Holmes et C.B. Tanner. 1954. Field capacity approximation based on the moisture transmitting properties of the soil. Proc. Soil Sci. Soc. Am. 18: 252-254.

Havey, M.J. et C.R. Grau. 1985. Decline of established alfalfa in soils naturally infested with Phytophthora megasperma f. sp. medicaginis and level of correlation by seedling assay. Plant Dis. 69: 221-224.

Jacob, J.-P., J.-N. Couture et M. Clément. 1989. Sol. Pages 55-82 in Plantes fourragères, culture. $2^{2}$ édition, AGDEX 120/20, Publication 02-8906, Conseil des productions végétales du Québec, Québec.

Knous, T.R. et J.E. Maxfield. 1976. Implications of metabolic squalene in alfalfa roots associated with Phytophthora root rot. Proc. Am. Phytopathol. Soc. 3: 239 (résumé)

Kuan, T.L. et D.C. Erwin. 1980a. Formae speciales differentiation of Phytophthora megasperma isolates from soybean and alfalfa. Phytopathology 70: 333338.

Kuan, T.L. et D.C. Erwin. 1980b. Predisposition effect of water saturation of soil on Phytophthora root rot of alfalfa. Phytopathology 70: 981-986.

Marks, G.C. et J.E. Mitchell. 1970. Detection, isolation, and pathogenicity of Phytophthora megasperma from soils and estimation of inoculum levels. Phytopathology 60: 1687-1690.
Matsumoto, N. et T. Sato. 1979. Phytophthora cryptogea Pethyb. \& Laff. found in alfalfa-field soils. Ann. Phytopathol. Soc. Jpn. 45: 362-368.

McKeen, W.E. et J. Traquair. 1980. Aphanomyces sp., an alfalfa pathogen in Ontario. Can. J. Plant Pathol. 2: 42-44.

Pratt, R.G. 1981. Morphology, pathogenicity, and host range of Phytophthora megasperma, $P$. erythrosepti$c a, P$. parasitica from arrowleaf clover. Phytopathology 71: 276-282.

Pratt, R.G. et J.E. Mitchell. 1973. Conditions affecting the detection of Phytophthora megasperma in soils of Wisconsin alfalfa fields. Phytopathology 63: 13741379.

Richard, C. 1985. Phytophthora root rot of alfalfa in Québec. Plant Dis. 69: 905.

Richard, C., A. Beghdadi et J.-G. Martin. 1991. Aphanomyces euteiches, a novel root pathogen to alfalfa in Québec. Plant Dis. 75: 319.

SAS Institute Inc. 1985a. SAS user's guide: basics. Version 5.0 edition. Cary, NC. 1290 pp.

SAS Institute Inc. 1985b. SAS user's guide: statistics. Version 5.0 edition. Cary, NC. 956 pp.

Schmitthenner, A.F. 1964. Prevalence and virulence of Phytophthora, Aphanomyces, Pythium, Rhizoctonia, and Fusarium isolated from diseased alfalfa seedlings. Phytopathology 54: 1012-1018. 